\title{
A Study on the Tensile Mechanical Properties of HSPE Twisted Rope
}

\author{
Jiangao Shi ${ }^{1}$, Wenwen $\mathrm{Yu}^{1}{ }^{1}$, Wenzhu Zhong ${ }^{2}$, Yongli Liu ${ }^{1}$, Lei Wang ${ }^{1}$, Xiaoxue \\ Chen ${ }^{1}$, Wen $\mathrm{Lu}^{3}$ and Jieming Zhai ${ }^{4}$, \\ ${ }^{1}$ East China Sea Fisheries Research Institute, Chinese Academy of Fishery Sciences, Shanghai \\ 200090, China \\ ${ }^{2}$ Fishery Machinery and Instrument Research Institute, Chinese Academy of Fishery Sciences, \\ Shanghai 200092, China \\ ${ }^{3}$ Pingyang Bihai Xianshan Seafood Development Co., Ltd., Zhejiang, 325400, China \\ ${ }^{4}$ Laizhou Minbo Aquatic Products Co., Ltd., Shandong, 261400, China
}

Keywords: high strength polyethylene fiber twisted rope, mechanical properties, fishing

Abstract. The tensile mechanical properties of the HSPE fiber twisted rope were studies. The result shows twist significantly impacts the breaking strength and related properties of HSPE fibers. When the 3-strand PA rope in a diameter $14 \mathrm{~mm}, 3$-strand PE monofilament rope in a diameter of $20 \mathrm{~mm}$, and 3-strand PP-PE monofilament rope in a diameter of $16 \mathrm{~mm}$ is replaced by the HSPE fiber twisted rope, the HSPE fibers rope breaking strength can be increased by $166.0 \% \sim 310.5 \%$, the raw material consumption can be reduced by $3.5 \% \sim 76.4 \%$, the rope diameter can be reduced by $0 \sim 50 \%$, the linear density can be reduced by $3.5 \% \sim 76.4 \%$, and the breaking elongation rate can be decreased by $87.0 \% \sim 89.0 \%$. The performance-price ratio, safety and tensile mechanical properties are favorable, and there is an extensive prospect of industrialized application in fisheries.

\section{Introduction}

Ropes, widely used in modern fishery, are usually made of common synthesized fibers such as polyethylene (PE) fiber, polypropylene (PP) fiber, polyamide (PA) fiber and polyester (PET) fiber, etc in a simple process. Common synthesized fibers, the basic structural unit of ropes, are usually made in the traditional process ${ }^{[1-7]}$. The tensile mechanical properties of rope directly determine the expansion, complication, offshore work specialization and safety against wind and wave of marine fisheries and projects ${ }^{[8-22]}$. The progress of modern fishery brings a higher demand for the safety, performance-price ratio, and tensile mechanical properties of the rope, and it urgently needs to carry out the research, development and industrialized application of new rope materials ${ }^{[7-18]}$. The high molecular weight polyethylene (UHMWPE) rope has outstanding comprehensive properties. At present, it has been applied in marine fishing and large aquatic farm fence. UHMWPE fiber mesh has played an important role in wave resistance of ocean fisheries, open sea net cage aquaculture, large deepwater net cage aquaculture and large aquaculture fencing ${ }^{[13-16]}$. However, UHMWPE rope is expensive now, and there are only a few researches on its performance-price ratio, physical properties and demonstrative application, which delay the generalized fishery application ${ }^{[5,9,11-22]}$. High performance but low price ropes are greatly demanded in modern fisheries, so some new fiber rope materials with a high performance-price ratio are chosen ${ }^{[1-23]}$. The technological progress of fiber material realizes the research on the tensile mechanical properties of new materials and the industrialized application of high strength ropes, and it has made some breakthroughs ${ }^{[20-22]}$. High 
strength polyethylene (HSPE) fiber twisted rope (we call it "HSPE fiber for fishing" differing from the other four types of high strength polyethylene fibers) with high breaking strength and performance-price ratio has gained wide attention. This paper analyzes and compares the tensile mechanical properties and performance-price ratio between HSPE fiber twisted ropes and common fiber ropes so as to provide scientific bases for the industrialized production and application of HSPE fiber twisted ropes.

\section{Materials and methods}

\section{Preparation of HSPE fiber for fishing}

The new material of HSPE fiber was prepared by Zhengzhou Zhongyuan Urethane Elastic Fiber Engineering Technology Co., Ltd.; 5 types of new materials of HSPE fiber (twisted in 0T/m, 10T/m, 20T/m, 30T/m, and 40T/m respectively) were prepared by the East China Sea Fisheries Research Institute via a twisting machine, and the fiber with the highest breaking strength was chosen as the basal fiber for the HSPE fiber twisted rope.

\section{Preparation of HSPE fiber twisted rope}

The HSPE fiber twisted rope in a diameter of $10 \mathrm{~mm}$ was made via ring twisting, strand combining, and rope making by using the HSPE fiber for fishing as the basal fiber. The common 3-strand PA rope, 3-strand PE rope and 4-strand twisted PE rope were made of the common synthesized fiber ${ }^{[2,}$ 23, 24]. Water soluble epoxy resin was used to impregnate, and the HSPE fiber twisted rope was impregnated into epoxy resin and cured ${ }^{[9-10]}$.

\section{Testing method}

Test instruments cover an electronic balance FA2004N, an electronic scale ACS-15A from China, a strength tester INSTRON-4466 (range $10 \mathrm{kN}$, resolution $0.1 \mathrm{~N}$ ) from Britain and a strength tester RHZ-1600 (range $1600 \mathrm{kN}$, resolution $0.08 \mathrm{kN}$ ) from Germany.

The test was carried out in a standard lab at $(20 \pm 3)^{\circ} \mathrm{C}$ and $\mathrm{RH}(65 \pm 5) \%$ constantly, and samples were balanced in the lab for $24 \mathrm{~h}$ above. The tensile mechanical properties of HSPE fiber were tested by the strength tester INSTRON-4466 at a drawing speed of $100 \mathrm{~mm} / \mathrm{min}$ and a sample length of $750 \mathrm{~mm}$. The tensile mechanical properties of HSPE fiber twisted rope were tested by the strength tester RHZ-1600 at a drawing speed of $100 \mathrm{~mm} / \mathrm{min}$. The tensile mechanical properties of HSPE fiber were tested according to SC/T $5005^{[25]}$. The tensile mechanical properties of HSPE fiber twisted rope were tested according to GB/T $8834^{[26]}$. Valid data obtained in the test were managed according to data processing specification of the corresponding standard.

\section{Results and Discussion}

\section{The effect of twist}

To analyze the effect of twist on the tensile mechanical properties of HSPE fiber, the tensile mechanical properties of 5 types of HSPE fiber in different twists were analyzed and compared (see Tab. 1), and the HSPE fiber with the highest breaking strength was chosen as the basal fiber of HSPE fiber twisted rope. 
Tab. 1 The tensile mechanical properties of HSPE fiber under different twist

\begin{tabular}{ccccc}
\hline NO. & $\begin{array}{c}\text { Linear density } \\
(\text { tex })\end{array}$ & $\begin{array}{c}\text { Twist } \\
(\mathrm{T} / \mathrm{m})\end{array}$ & $\begin{array}{c}\text { Breaking strength (cN/dtex) } \\
\text { Knot strength } \\
(\mathrm{cN} / \mathrm{dtex})\end{array}$ \\
\hline 1 & 1222 & 0 & 19.58 & 7.06 \\
2 & 1222 & 10 & 20.55 & 6.62 \\
3 & 1222 & 20 & 21.96 & 5.91 \\
4 & 1222 & 30 & 20.29 & 6.17 \\
5 & 1222 & 40 & 20.02 & 8.47 \\
\hline
\end{tabular}

Tab. 1 shows that the HSPE fiber in the twist of $20 \mathrm{~T} / \mathrm{m}$ is the best and integrated with the HSPE fibers, and it is used as the basal fiber to make the HSPE fiber twisted rope. The breaking strength and knot breaking strength of the HSPE fiber for fishing were $21.96 \mathrm{cN} / \mathrm{dtex}$ and 5.91 cN/dtex respectively, $322.3 \%$ and $64.2 \%$ higher than the indexes for the fishing PE monofilament according to the industrial standard SC/T 5005. The internal molecular orientation of the HSPE fiber for fishing was modified by the special synthesizing process, and thus the crystallinity and orientation degree of the HSPE fiber for fishing were increased, and the fiber was given higher breaking strength and knot breaking strength ${ }^{[1-7]}$. Tab. 2 compares the tensile mechanical properties between the HSPE fiber for fishing and common synthesized fibers.

Tab. 2 Compares the tensile mechanical properties between the HSPE fiber for fishing and common synthesized fibers

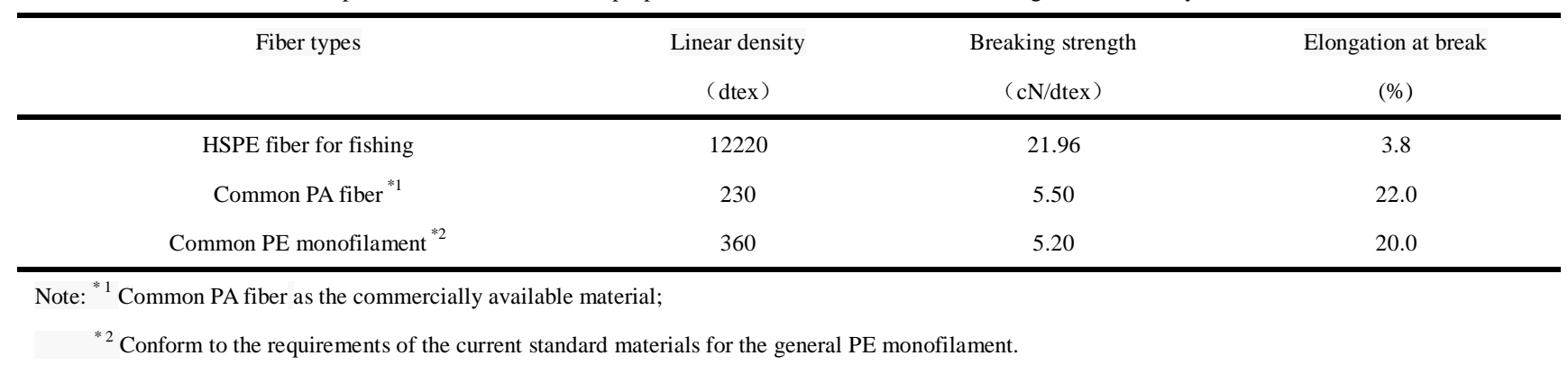

High breaking strength is one of the most important properties of the HSPE fiber for fishing. Comparing with common synthesized fibers, the HSPE fiber for fishing has a distinctly superior breaking strength which is $299.3 \%$ and $322.3 \%$ higher than the common PA fiber and common PE monofilament respectively. Elongation is a key factor in the material characteristics and the application properties, and the low elongation rate is another important property for the HSPE fiber for fishing; comparing with the common PE monofilament, the high molecular chain of HSPE fiber for fishing has a low elongation deformation rate in drawing (being elongated or drifted), and the elongation rate of the HSPE fiber for fishing is lower than common synthesized fibers ${ }^{[1-7]}$. The breaking elongation rate of the HSPE fiber for fishing is $3.8 \%$, reduced by $82.7 \%$ and $81.0 \%$ than the common PA fiber and the common PE monofilament.

\section{The tensile mechanical properties of HSPE fiber twisted rope}

The HSPE fiber twisted rope and HSPE fiber composite rope in diameters of $10 \mathrm{~mm}$ were made by using the HSPE fiber for fishing as the basal fiber, and the comparison of test results of tensile mechanical properties between the ropes and common synthesized ropes are shown in Tab. 3 . 
Tab. 3 comparison of the tensile mechanical properties HSPE fiber twisted rope and ordinary PE synthetic fiber rope

\begin{tabular}{|c|c|c|c|c|c|c|}
\hline \multirow[t]{2}{*}{ Rope types } & Diameter & Linear density & Breaking Load & $\begin{array}{l}\text { Breaking } \\
\text { Strength }\end{array}$ & Elongation & \multirow[t]{2}{*}{$\begin{array}{c}\text { Price } \\
(\mathrm{RMB} / \mathrm{kg})\end{array}$} \\
\hline & $(\mathrm{mm})$ & $($ ktex $)$ & $(\mathrm{kN})$ & $(\mathrm{cN} / \mathrm{dtex})$ & $(\%)$ & \\
\hline HSPE fiber twisted rope & 10 & 47.3 & 31.47 & 6.65 & $5.0 \sim 8.0$ & 40.0 \\
\hline Acceptable product of 3 -strand PA rope $\mathrm{e}^{[2,23]}$ & 10 & 62.0 & 13.90 & 2.24 & $55 \sim 63$ & 34.5 \\
\hline Acceptable product of 3 -strand PE rope $\mathrm{e}^{[2,23]}$ & 10 & 49.0 & 8.85 & 1.81 & $45 \sim 55$ & 16.5 \\
\hline 4-strand twisted PE rope ${ }^{[2,26]}$ & 10 & 50.1 & 9.81 & 1.96 & $45 \sim 55$ & 16.5 \\
\hline Acceptable product of 3 -strand PA rope $\mathrm{e}^{[2,23]}$ & 14 & 122 & 27.30 & 2.24 & $55 \sim 63$ & 34.5 \\
\hline Acceptable product of 3 -strand PE rope $\mathrm{e}^{[2,23]}$ & 20 & 200 & 32.30 & 1.62 & $45 \sim 55$ & 16.5 \\
\hline Acceptable product of 3 -strand PP-PE rope $\mathrm{e}^{[2,23]}$ & 16 & 115 & 28.80 & 2.50 & $45 \sim 55$ & 16.5 \\
\hline HSPE fiber composite twisted rope & 10 & 50.1 & 34.89 & 6.96 & $6.0 \sim 9.0$ & 36.4 \\
\hline
\end{tabular}

\section{The effect of resin treatment}

Resin treatment distinctly impacts the tensile mechanical properties, wear resistance and aging resistance of the rope ${ }^{[9-10]}$. To further improve the comprehensive performance of the HSPE fiber twisted rope, it is significantly necessary to analyze and study the effect of resin treatment on the tensile mechanical properties of the HSPE fiber twisted rope. The HSPE fiber twisted rope was made into the HSPE fiber composite twisted rope via resin impregnation and cure, etc, as a result, the internal fiver was more close, rope fluff was reduced, the tensile force in preparing the composite twisted rope was uniformly transmitted to the fiber, and thus the breaking load and breaking strength of the composite twisted rope were improved. Compared with the HSPE fiber twisted rope before resin treatment, the linear density of the HSPE fiber composite twisted rope was improved by $5.9 \%$, the breaking load was improved by $10.9 \%$, the breaking strength was improved by $4.7 \%$, and the breaking elongation rate was improved by $15.4 \%$, see Tab. 3$)^{[9-10]}$.

\section{Analysis on the breaking load and energy saving and consumption reducing effect of HSPE fiber twisted rope}

The rope breaking load is mainly determined by the after-treatment process, basal fiber material, rope yarn load utilization rate and rope making process (e.g. rope yarn twist, rope yarn arrangement, rope length of lay and number of rope strands), etc ${ }^{[2,8-22]}$. Tab. 3 shows that the HSPE fiber twisted rope has a superior breaking load than common synthesized fiber ropes, the breaking load is improved by $126.4 \%, 255.6 \%$ and $220.8 \%$, the linear density is reduced by $23.7 \%, 3.5 \%$ and $5.6 \%$, and the raw material consumption is reduced by $23.7 \%, 3.5 \%$ and $5.6 \%$ respectively if the HSPE fiber twisted rope in a diameter of $10 \mathrm{~mm}$ is used to replace the 3-strand PA rope, 3-strand PE monofilament rope and 4-strand twisted PE rope. The breaking load is improved by $15.3 \%$, the linear density is reduced by $61.2 \%$, the diameter is reduced by $28.6 \%$, the raw material consumption is reduced by $61.2 \%$, and the netting gear resistance in water is reduced correspondingly if the HSPE fiber twisted rope in a diameter of $10 \mathrm{~mm}$ is used to replace the 3 -strand PA rope in a diameter of $14 \mathrm{~mm}$; the breaking load is improved by $2.6 \%$, the linear density is reduced by $76.4 \%$, the diameter is reduced by $50.0 \%$, the raw material consumption is reduced by $76.4 \%$, and the netting gear resistance in water is reduced correspondingly if the HSPE fiber twisted rope in a diameter of $10 \mathrm{~mm}$ is used to replace the 4-strand twisted PE rope in a diameter of $20 \mathrm{~mm}$; the breaking load is improved by $9.3 \%$, the linear density is reduced by $58.9 \%$, the diameter is reduced by $37.5 \%$, the raw material consumption is reduced by $58.9 \%$, and the netting gear resistance in water is reduced correspondingly if the HSPE fiber twisted rope in a diameter of $10 \mathrm{~mm}$ is used to replace the 
3-strand PP-PE monofilament rope in a diameter of 16mm; therefore, comparing with the common synthesized fiber ropes in Tab. 3, the HSPE fiber twisted rope has a superior breaking load and a distinct effect in reducing the consumption and resistance. In fishery production without degrading the rope breaking load, people can use the HSPE fiber twisted rope in a diameter of $10 \mathrm{~mm}$ to replace the 3-strand PA rope in a diameter of $14 \mathrm{~mm}, 3$ strand $\mathrm{PE}$ monofilament rope in a diameter of $20 \mathrm{~mm}$, and 3-strand PP-PE monofilament rope in a diameter of $16 \mathrm{~mm}$ as the fishing rope to improve the rope load and netting gear safety, reduce the netting gear resistance in water, reduce the rope linear density and raw material consumption, realize the energy saving and consumption reducing, and promote the fishery emission control and marine food supply.

\section{Analysis on the breaking strength and elongation of HSPE fiber twisted rope}

Tab. 3 shows that the HSPE fiber twisted rope has higher breaking strength and lower elongation rate than common synthesized fiber ropes. The breaking strength is improved by $196.9 \%, 267.4 \%$ and $239.3 \%$ and the elongation rate was reduced by $89.0 \%, 87.0 \%$ and $87.0 \%$ when the 3 -strand PA rope, 3-strand PE monofilament rope and 4-strand twisted PE rope in the diameter of $10 \mathrm{~mm}$ is replaced by the HSPE fiber twisted rope in the same diameter. The breaking strength is improved by $196.9 \%, 310.5 \%$ and $166.0 \%$ and the elongation rate was reduced by $89.0 \%, 87.0 \%$ and $87.0 \%$ when the 3-strand PA rope in a diameter of $14 \mathrm{~mm}$, 3-strand PE monofilament rope in a diameter of $20 \mathrm{~mm}$ and 3-strand twisted PP-PE monofilament rope in a diameter of $16 \mathrm{~mm}$ is replaced by the HSPE fiber twisted rope in a diameter of $10 \mathrm{~mm}$. The distinct differences of breaking strength and elongation between the HSPE fiber twisted rope and common synthesized fiber ropes are mainly caused by the different tensile mechanical properties of the basal fibers. The HSPE fiber twisted ropes made of the basal fiber of HSPE fiber for fishing with a breaking strength of $21.96 \mathrm{cN} / \mathrm{dtex}$ and an elongation rate of $3.8 \%$ while the 3 -strand PA rope is made of common PA fiber with a breaking strength of $5.50 \mathrm{cN} / \mathrm{dtex}$ and an elongation rate of $22.0 \%$, the breaking strength of common PE monofilament is $5.20 \mathrm{cN} / \mathrm{dtex}$ and the breaking elongation is $20.0 \%$ (Tab. 2), so there is a distinct difference of tensile mechanical properties between the HSPE fiber for fishing and common synthesized fibers. High breaking strength and low elongation are important features for the HSPE fiber for fishing differing from common synthesized fibers. The internal molecular structure of the HSPE fiber for fishing is modified by the special synthesizing process, the molecular chain is arranged more closely, and the chain stretch is inhibited, so this HSPE fiber for fishing in a specific aggregative structure is given a higher breaking strength and a low elongation, and the HSPE fiber for fishing rope twisted by it has a higher breaking strength and a lower elongation than common synthesized fiber ropes (Tab. 3) ${ }^{[2,8-22]}$. Comparing with the HSPE fiber for fishing, the molecular interacting forces of common synthesized fibers in Tab. 2 are small, and the elongation strains are large, so the common synthesized fiber ropes are shaped with low breaking strengths and larger elongations (Tab. 1) ${ }^{[1-7]}$.

The rope prices of HSPE fiber twisted rope in the current market in Tab. 3. When the 3-strand $\mathrm{PA}$ ropes in diameters of $10 \mathrm{~mm}$ and $14 \mathrm{~mm}$ and conforming to GB/T 18674 are replaced by the HSPE fiber for fishing twisted rope in a diameter of $10 \mathrm{~mm}$ and same lengths for the fishing rope, the rope unit prices are increased by $15.9 \%$ and $15.9 \%$ respectively, but the weights are reduced by $23.7 \%$ and $61.2 \%$, so the rope costs are reduced by $11.5 \%$ and $55.0 \%$.

\section{Analysis on the safety of HSPE fiber rope}

Comparing with common synthesized fiber ropes (3-strand PA rope, 3-strand PE monofilament rope, 4-strand twisted PE rope and 3-strand PP-PE monofilament rope) in diameters of $10 \mathrm{~mm} 20 \mathrm{~mm}$ in Tab. 3, the breaking load of high-strength PE fiber twisted rope is improved by $2.6 \% \sim 255.6 \%$, and 
the breaking strength is improved by $166.0 \%$ 310.5\%, therefore, the fishing rope breaking load and breaking strength and the fishery safety can be effectively improved by replacing the common synthesized fiber ropes in diameters of $10 \mathrm{~mm} 20 \mathrm{~mm}$ in Tab. 3 with the HSPE fiber twisted

rope ${ }^{[2-6,8-22]}$. Similar with other high strength synthesized fiber ropes, the elongation disadvantage of high PE fiber twisted rope is made up and the demand for impact load of fishing rope is satisfied by the high strength advantage; a greater energy can be absorbed by the high strength PE split-film twisted rope and only a little rebounding force will be generated at rope breaking, the accidental injury to fishers and aquaculture farm owners caused by rope breaking can be highly reduced, and thus the fishery safety can be highly improved.

\section{Conclusion}

The HSPE fiber rope was prepared and the tensile mechanical properties of the HSPE fiber twisted rope were studies. The result shows twist significantly impacts the breaking strength and related properties of HSPE fibers. The HSPE fibers rope breaking strength can be increased by $166.0 \% \sim 310.5 \%$, the raw material consumption can be reduced by $3.5 \% \sim 76.4 \%$, the rope diameter can be reduced by $0 \sim 50 \%$, the linear density can be reduced by $3.5 \% \sim 76.4 \%$, and the breaking elongation rate can be decreased by $87.0 \%$ 89.0\% if the 3-strand PA rope in a diameter $14 \mathrm{~mm}$, 3 -strand PE monofilament rope in a diameter of 20mm, and 3-strand PP-PE monofilament rope in a diameter of $16 \mathrm{~mm}$ is replaced by the HSPE fiber twisted rope without modifying the rope breaking load advantage; the performance-price ratio, safety and tensile mechanical properties are favorable, and there is an extensive prospect of industrialized application in fisheries.

\section{Acknowledgement}

This research was supported by the National Key Technology R\&D Program (2013BAD13B03, 2013BAD13B02), 2013 Marine Economic Innovation Demonstration Project “650t Annual Yield of Daiquzu Large Yellow Croaker in Offshore Deep Water Cages”.

\section{References}

[1] M.C. Sun, X.R. Zou, and J. Zhang, et al. : Marine Fishery Technology (China Agriculture Press Beijing 2012).

[2] M.C. Sun, J.G. Shi and C.C. Xu, et al.: Fishery Material and Technology (China Agriculture Press, Beijing 2009).

[3] J.G. Shi, L.M. Wang and X.Z. Chen, et al. : Fishing Nets and Anti-contamination Technologies, (Donghua University Press, Shanghai 2011).

[4] J.Z. Xu: Deep water cages and barrier net aquiculture (China Agriculture Press, Beijing 2007).

[5] L.M. Wang: Journal of Fisheries of China Vol 24 (2000), P. 480.

[6] J.G. Shi, L.M. Wang and X.L Chen, et al: Journal of Modern Fisheries Information Vol 23 (2008), P. 9.

[7] K. Zhang: Polymer Physics (Chemical Industry Press, Beijing 1981).

[8] M.C. Sun, J. Zhang, and W.G. Qian, et al: Selectivity of Fishing Gear (China Agriculture Press, Beijing 2004).

[9] H.Y. Ma, Y.P. Guo and L.S. Mao: Marine Fisheries Vol 27 (2005), P. 154.

[10] J. Liu, Y. Wang and C. Wang, et al.: Science \& Technology in Chemical Industry Vol 10 (2002), P. 6. 
[11] J.G. Shi, Y.L. Liu and L.M. Wang, et al.: Modern Fisheries Information Vol 28 (2013), P. 127.

[12] J.G. Shi, L.M. Wang and X.L. Chen, et al.: Journal of Modern Fisheries Information Vol 11 (2010), P. 17.

[13] J.G. Shi, Y.L. Liu and H.Y. Ma, et al.: Journal of Modern Fisheries Information Vol 12 (2010), P. 16.

[14] Anon: Fishing News International Vol 39 (2000), P. 34.

[15] Anon: Offshore Vol 72 (2012), P. 90.

[16] Anon: Fishing News International Vol 33 (1994), P. 14.

[17] D.Z. Wo: Composite Materials (Chemical Industry Press, Beijing 2000).

[18] Y.P. Guo, H.Y. Ma, L.S. Mao: Journal of Fisheries of China Vol 29 (2005), P. 724.

[19] J.G. Shi and L.M. Wang. Journal of Shanghai Ocean University Vol 12 (2003), P. 371.

[20] J.G. Shi, W. Yu and Y. Liu, et al.: Advances in Engineering Research Vol 41 (2015), P. 356.

[21] J.G. Shi, W. Yu and W.Z. Zhong, et al.: Advanced Materials Research Vol 1033-1034 (2014), P. 921-926.

[22] W. Yu, J.G. Shi, and W.Z. Zhong, et al.: Applied Mechanics and Materials Vol 633-634 (2014), P. 221.

[23] GB/T 18674-2002 General technical specifications for ropes for fisheries (China Press of Standards, Beijing 2002).

[24] ISO 1969: 2004 Fiber ropes - Polyethylene - 3- and 4-strand ropes (Switzerland, 2004).

[25] SC/T 5005-2014 Polyethylene Monofilament for fisheries (China Press of Standards, Beijing 2014).

[26] GB/T 8834-2006 Ropes - Determination of certain physical and mechanical properties (China Press of Standards, Beijing 2006). 